Supporting Information for

\title{
Observation of Single Tin Dioxide Nanoribbons by Confocal \\ Raman Microspectroscopy
}

Fengping Wang, Xingtai Zhou, Jigang Zhou, Tsun-Kong Sham ${ }^{*}$ and Zhifeng Ding*

Department of Chemistry, The University of Western Ontario, London, ON N6A 5B7

Canada

e-mails: tsham@uwo.ca (TKS); zfding@uwo.ca (ZFD)

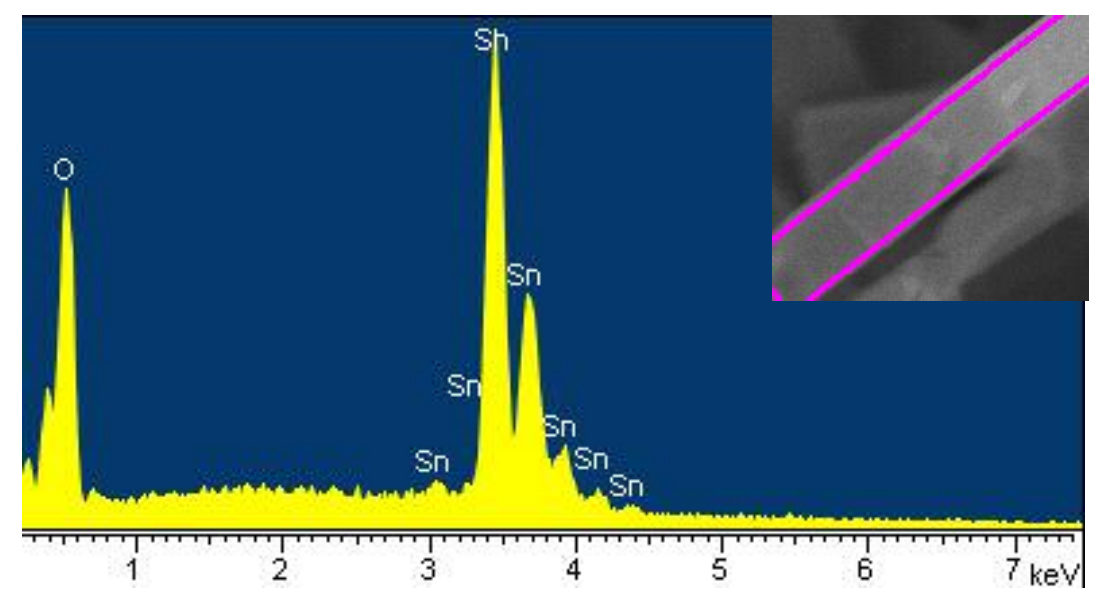

Standard :

O $\quad \mathrm{SiO} 2$ 1-Jun-1999 12:00 AM

Sn $\quad$ Sn 1-Jun-1999 12:00 AM

\begin{tabular}{l|ll}
\hline Element & Weight $\%$ & Atomic\% \\
& & \\
O K & 22.42 & 68.19 \\
Sn L & 77.58 & 31.81 \\
& & \\
Totals & 100.00 &
\end{tabular}

Fig. S1a 


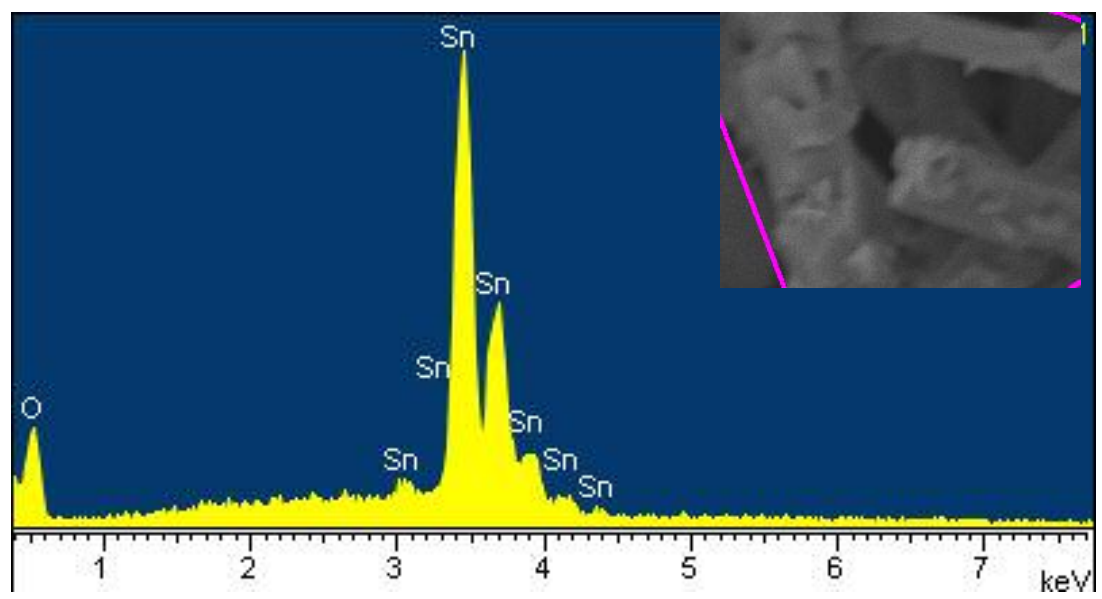

Standard :

$\begin{array}{lcc}\mathrm{O} & \mathrm{SiO} 2 & \text { 1-Jun-1999 12:00 AM } \\ \mathrm{Sn} & \mathrm{Sn} & \text { 1-Jun-1999 12:00 AM }\end{array}$

\begin{tabular}{l|ll}
\hline Element & Weight $\%$ & Atomic\% \\
& & \\
O K & 8.59 & 41.07 \\
Sn L & 91.41 & 58.93 \\
& & \\
Totals & 100.00 & \\
\hline
\end{tabular}

Fig. S1b

Fig.S1. The elemental composition of the two types of the ribbon surfaces was analyzed by EDX. The ratio of Sn to oxygen is 1:2 for the nanoribbons with a smooth surface in Fig. S1a. However, there is oxygen deficiency in the second type of ribbon surfaces, where the Sn:O ratio is almost 1.43:1 in Fig. S1b. 


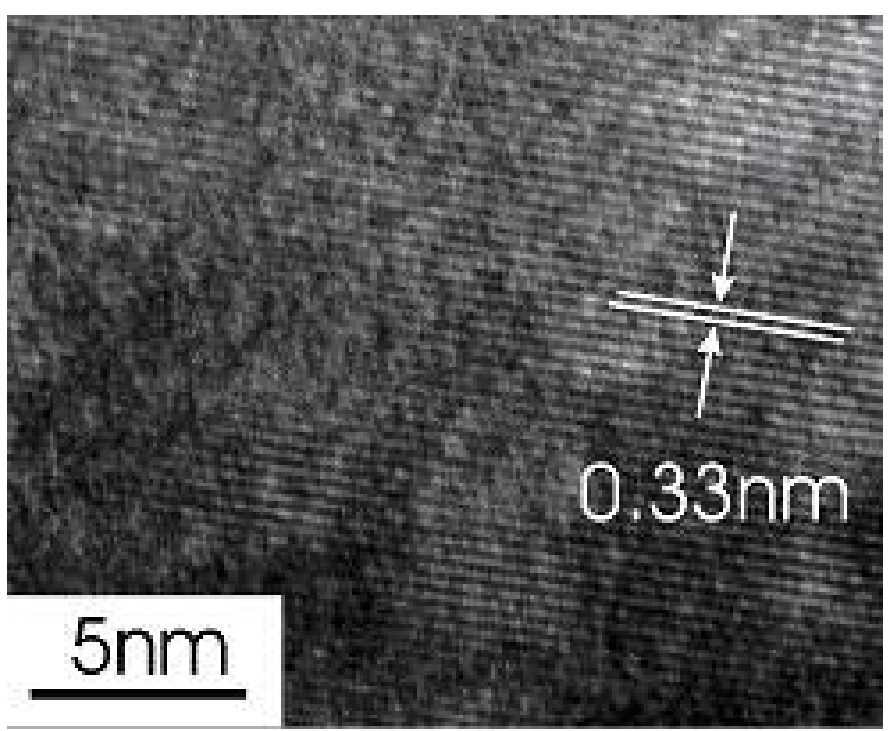

Fig. S2. A typical high-resolution TEM image of a flat $\mathrm{SnO}_{2}$ nanoribbon 


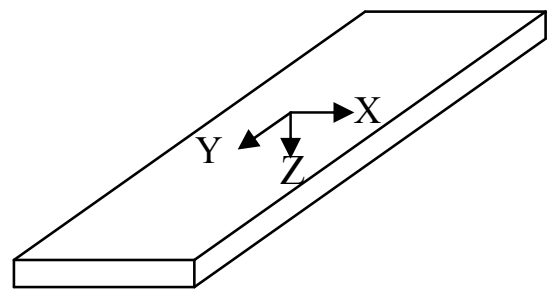

Fig. S3a

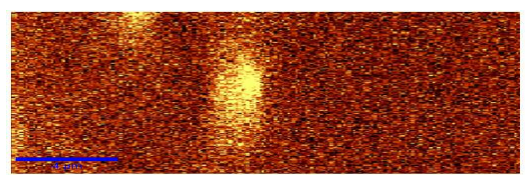

Fig. S3b

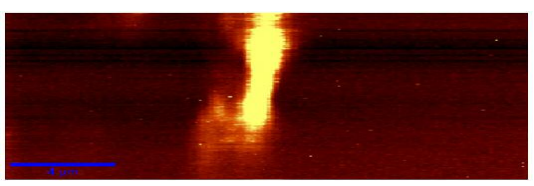

Fig. S3c

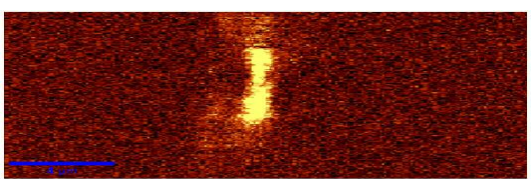

Fig.S3d

Fig.S3. The depth scan Raman images of a single nanoribbon. Fig. S3a is a schematic of the ribbon for the depth scan where $\mathrm{X}$ and $\mathrm{Y}$ represent the dimensions on the sample surface, and $\mathrm{Z}$ is the normal direction, and coordinate origin is on the center of the image, the depth scan is along the $\mathrm{X}-\mathrm{Z}$ coordinate plane; Fig. S3b was constructed from the integration of the normal Raman band of $\mathrm{SnO}_{2}$ at $633 \mathrm{~cm}^{-1}$; the image shown in Fig.S3c was created from the extra signal bands between 1000 and $1800 \mathrm{~cm}^{-1}$; the image shown in Fig.S3d was built from the double peak at 238 and $245 \mathrm{~cm}^{-1}$. The blue scale bar is $10 \mu \mathrm{m}$, representing the dimensions on the sample surface and the normal direction, respectively. The yellow bright color demonstrates the stronger intensity of the Raman band whereas the dark brown illustrates the weaker intensity. 

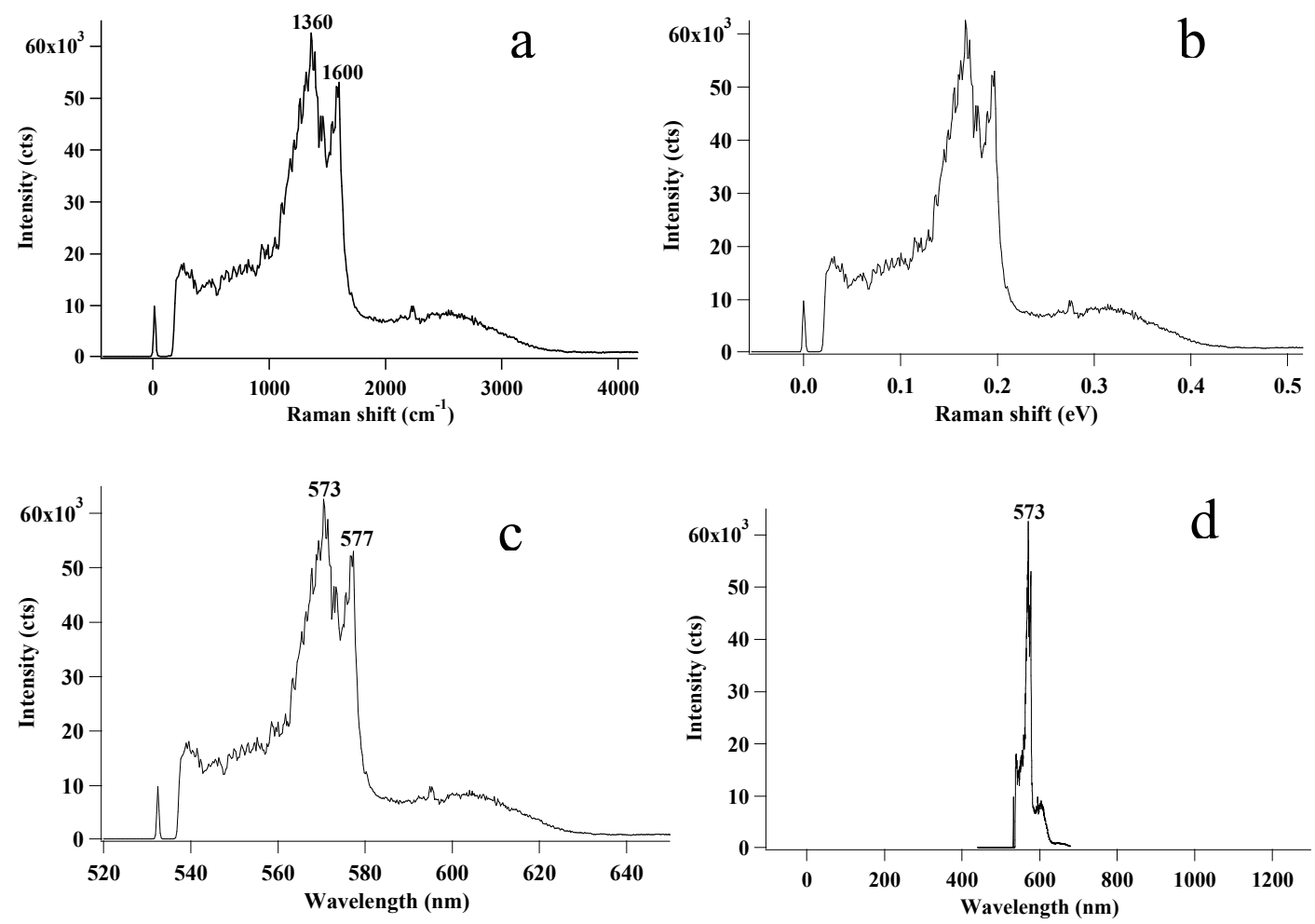

Fig. S4. The integration spectrum $(t=10$ second $)$ plotted by using different kind of Raman shift units. Fig. S4a was plotted according to intensity versus Raman shift in wave number; Fig. S4b was plotted according to intensity versus Raman shift in electron volt; Figs. S4c and S4d were plotted according to intensity versus Raman shift in nanometer. It is observed that the many small peaks considered as noise in the paper disappear with larger Raman shift scales, where only two clear peaks can be observed; Furthermore, the whole width of the extra band as shown in Fig. S3d is really narrow compared to photoluminescence peaks. 